Abstract

\title{
Phytochemical and Pharmacological Study of Plectranthus ecklonii Benth ${ }^{\dagger}$
}

\author{
Eva María Domínguez-Martín 1,2, , Ana Margarida Antão 1,‡, Epole Ntungwe 1,2, Vera M. S. Isca 1,3, \\ Ana María Díaz-Lanza ${ }^{2}$ and Patrícia Rijo ${ }^{1,3, *}$
}

Citation: Domínguez-Martín, E.M.; Antão, A.M.; Ntungwe, E.; Coimbra, R.; Isca, M.S.V.; Díaz-Lanza, A.M.; Rijo, P. Phytochemical and Pharmacological Study of Plectranthus ecklonii Benth. Proceedings 2021, 79, 8. https://doi.org/10.3390/IECBM202008567

Published: 30 November 2020

Publisher's Note: MDPI stays neutral with regard to jurisdictional claims in published maps and institutional affiliations.

Copyright: $₫ 2020$ by the authors. Licensee MDPI, Basel, Switzerland. This article is an open access article distributed under the terms and conditions of the Creative Commons Attribution (CC BY) license (http://creativecommons.org/licenses/by/4.0/).
1 CBIOS-Research Center for Biosciences \& Health Technologies, Universidade Lusófona de Humanidades e Tecnologias, Campo Grande 376, 1749-024 Lisboa, Portugal; evam.dominguez@uah.es (E.M.D.-M.); ana.antao88@gmail.com (A.M.A.); p5999@ulusofona.pt (E.N.); veraisca@msn.com (V.M.S.I.)

2 Department of Biomedical Sciences, Faculty of Pharmacy, University of Alcalá de Henares, Ctra. A2, Km 33.100-Campus Universitario, 28805 Alcalá de Henares, Madrid, Spain; ana.diaz@uah.es

3 Instituto de Investigação do Medicamento (iMed.ULisboa), Faculdade de Farmácia, Universidade de Lisboa, 1649-003 Lisboa, Portugal

* Correspondence: patricia.rijo@ulusofona.pt

+ Presented at the 1st International Electronic Conference on Biomolecules: Natural and Bio-Inspired Therapeutics for Human Diseases, 1-13 December 2020; Available online: https://iecbm2020.sciforum.net/. $\ddagger$ Authors contributed equally to this work.

Abstract: The use of herbal products for the treatment, prevention and cure of diseases is one of the oldest human medicinal practices. In fact, the majority of the population in developing countries depend on ancestral plant knowledge for healthcare. However, there is still a gap between progress observed in clinical pharmacy and in the field of herbal and traditional medicine, as there remain many natural products with biological activity to be identified. Plectranthus species (Lamiaceae family) have a widespread ethnobotanical use and are often cited by its medicinal properties and application, particularly in folk medicine. They contain many antioxidant compounds and exhibit several effects (anti-inflammatory, antimicrobial and antifungal) which suggest that Plectranthus may be a promising genus for the discovery of medicinal compounds. Thus, the isolation of secondary metabolite compounds from the Plectranthus spp. is important to scientifically validate the popular uses of these plants and to find new sources of potentially economically important products or compounds, which can be transformed into active pharmaceutical ingredients. Furthermore, the cytotoxicity evaluation of the plant extracts and their active ingredients are required for their effective and safe therapeutic use. This work enumerates the compounds isolated to date from Plectranthus ecklonii Benth., extracts and their biological activities. The HPLC analysis presented is part of an ongoing project at CBIOS of identification, quantification and evaluation of the bioactive components (in particular, diterpenes and hydrocinnamic acids) in different species of Plectranthus.

Keywords: Plectranthus; Plectranthus ecklonii; diterpenes; abietanes; parviflorons; bioactivity; antioxidant; antimicrobial; anti-inflammatory; anti-tumoral

Institutional Review Board Statement: Not applicable.

Informed Consent Statement: Not applicable.

Data Availability Statement: Data sharing not applicable.

Acknowledgments: This research was funded by Fundação para a Ciência e a Tecnologia (FCT, Portugal), through projects UIDP/04567/2020 and UIDB/04567/2020. E.M.D.-M gratefully acknowledges being the recipient of a predoctoral FPU 2019 fellowship from the University of Alcalá de Henares. 